\title{
Research of Fault Diagnosis of Wavelet Analysis for Asynchronous Motor
}

\author{
Fei Zhou ${ }^{1, \text { a }}$ \\ 1Xijing University, \\ Shaanxi Xi'an ,China \\ aE-mail:743040357@qq.com \\ Guomin Lin ${ }^{2, d}$ \\ 2Xijing University, \\ Shaanxi Xi'an ,China \\ bE-mail:641922530@qq.com
}

\author{
Miao Shang ${ }^{3, d}$ \\ 3 Xijing University, \\ Shaanxi Xi'an ,China \\ c E-mail: 445700839@qq.com \\ Zhengqiang $\mathrm{Li}^{4, \mathrm{~d}}$ \\ 4 Xijing University, \\ Shaanxi Xi'an ,China \\ d E-mail: 553187250@qq.com
}

\begin{abstract}
In this article,the working principle of the asynchronous motor and its common failure forms are analyzed and summarized.On this basis, the wavelet change of the application of the asynchronous motor that is carried out 。 In normal state and failure state, the motor vibration and current signal was reconstruct of the wavelet packet decomposition and single, and the fault characteristic frequency band that is conducted to extract.The characteristics of the normal operation and fault run time spectrum is carried on the detailed comparison. Based on the wavelet packet decomposition and reconstruction of motor fault feature extraction method can effective characterization of failure from the characteristic spectrum reconstruction signal waveform 。 Thus proves that the method based on wavelet analysis can accurately implement motor fault diagnosis.
\end{abstract}

Key words-asynchronous motor; wavelet analysis; fault form; fault diagnosis; wavelet algorithm

\section{INTRODUCTION}

Asynchronous motor is important rotating machinery in power system, its operation condition has a great influence for the safe operation of power system. When an asynchronous motor internal early failure after a certain amount of time and become a sudden failure, the existing relay protection device can only be the fault motor removal from the power grid, to prevent further failure[1]. But electricity production will still be interrupted by accident, expensive motor will be severely damaged. Therefore, studying how the early failure of the asynchronous motor on-line detected in a timely manner, it is of great social benefits and economic benefits. In asynchronous motor fault signal, the non-stationary signal is often include. Such as the transition process when starting, current mutation signal and vibration signal of bearing failure caused by complex failure occurs when the stator winding and so on. Analysis method of the traditional Fourier transform is a lot of limitations in extract fault characteristics, creating a higher miscarriage rate will be generated [2]. Wavelet transform has excellent time-frequency localization property, and is suitable for the analysis of the unsteady signal. Based on the idea of wavelet transform was introduced to the early fault detection of the asynchronous motor. Asynchronous motor rotor, stator and bearing early faults detection method was studied, and a set of early malfunction of asynchronous motor wavelet intelligent diagnosis system is developed.

\section{WORKING PRINCIPLE AND COMMON FAILURE FORMS OF THE ASYNCHRONOUS MOTOR}

\section{A. Working principle of the asynchronous motor}

Asynchronous motor is mainly composed of stator and rotor of two parts, the air gap between them. It is by the rotating stator magnetic field cutting rotor conductors induced current, the rotating magnetic field effect on the rotor induced current and drive the rotor rotation, so as to realize the conversion of electrical energy [3].

When the symmetric three-phase motor stator winding into the order when the three phase current, to produce a rotating magnetic field inside the motor. Set an instantaneous current and the magnetic field between as shown in figure 1-1, and n. speed clockwise. Due to the relative motion between the rotor conductors and rotating magnetic field and the induced electromotive force in the rotor conductors. If the rotating magnetic field as static, the equivalent of the rotor conductor counterclockwise cutting lines, right-hand rule to determine the direction of induction electromotive force is available. Because the rotor winding is closed.

So with same direction induction electromotive force induced current is produced. In this way, the rotor on the upper conductor of electric current from a paper out, is at the lower flow in.

Electricity (current carrying conductor in magnetic field by electromagnetic force, so the rotor conductors carrying induced current interaction with a rotating magnetic field to produce electromagnetic force $\mathrm{F}$, can use left hand rule to judge its direction [4]. Form a rotating magnetic field and the force of the rotation direction of the electromagnetic torque, the rotor along the direction of the 
rotating magnetic field with $\mathrm{n}$ the speed of rotation. Asynchronous motor rotor rotational speed $\mathrm{n}$ always lower than the speed of the rotating magnetic field $n$.

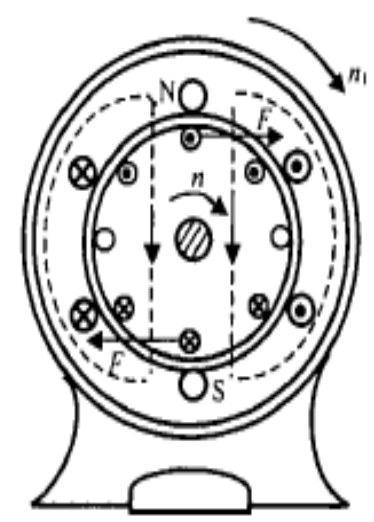

Figure 1. The Asynchronous rotation schematic diagram

\section{B. Common malfunction forms of asynchronous motor}

The type of the asynchronous motor fault depends on the structure and the working environment of machine. No matter what kind of failure, can according to certain development mode or mechanism, that is, from the initial defects become a part of the fault, or eventually led to the completely damaged. Time needed for the development process of each are not identical, because of the specific situation is different and there is a difference [5]. However, the most important thing is, no matter what kind of failure will have its early signs. Through the development of the monitoring can find some basic fault, thus the recognition of the early signs of failure [6].

(1) The stator core fault. Asynchronous motor stator core structure is: from the back of the core silicon steel sheet clamped, and then fixed on the stator's support. If in the process of manufacturing or repair damage to the stator core in rotor, can make the stator formed between the short circuit.

(2) The winding insulation failure. Insulation system is intrinsic to the motor, mechanical or electrical is one of the most weak part. Winding fault is often due to the insulation defect formation. 3 the wavelet transform.

(3) The failure of the stator of end winding. In the process of the motor running, under the action of stator end windings in Rio, mobile phenomenon happens. When the support structure relaxation, will end winding down.

(4) The fault of rotor winding. For squirrel cage induction motor, the rotor fault is often characterized by the following several ways: rotor broken bar end ring is broken, and casting in the cast aluminum rotor clearance and bubbles.

(5) The rotor fault of ontology. Fault general performance for the unbalanced rotor ontology, in the wrong, shaft bending, and eccentric shaft crack, etc.

(6) Electric imbalance fault. Electric imbalance fault mainly have three phase power supply imbalance, motor open phase running.

(7) Bearing failure. The motor rotor on both ends of before and after is supported by bearings. Failure form is usually characterized by wear, pitting, inside and outside the ring fracture failure. Bearing fault of oscillations, lead to excessive vibration, is a common vibration source of the motor.

\section{WAVELET ANALYSIS TEST METHOD OF ASYNCHRONOUS MOTOR}

Basic principle of wavelet transform in the paper, on the basis of wavelet transform time-frequency window characteristics was analyzed. From the perspective of timefrequency window, the selection of wavelet function should be followed by some common principles have been proposed in the practical application. Commonly used wavelet function of amplitude-frequency characteristic and phase frequency characteristics were analyzed from the Angle of the frequency domain. The characteristics of asynchronous motor rotor fault characteristic were studied in the process of starting. The wavelet ridge line of rotor broken bar fault detection method is proposed in the process of starting, and realizes the accurate diagnosis of faults in rotor asynchronous motor starting process.Unsteady signal is often included in the current signal of faulted synchronous motor, such as the start-up period, the sudden change signal of when faults happen in stator, and complicated vibration signals of faulted motor axis, etc. The traditional analysis method of Fourier transform is suited to process steady signal, but restricted for unsteady signal. Employing Fourier transform for analyzing unsteady signals, big error or even serious faulted diagnosis may occur. However, wavelet transform has excellent time-frequency localization ability, and very suited to do the analysis of unsteady signals. Based on this thinking, wavelet transform is introduced in this dissertation to detect the incipient faults of asynchronous motor. The research work mainly focuses on the wavelet analysis detection method the incipient faults of rotor, stator and axis, and as an actual fruit, a set of wavelet intelligent diagnosis system of incipient faults of asynchronous motor has been developed [7]. On the basis of introducing the basic theory of wavelet transform, the characteristic of time-frequency window is deeply studied in this dissertation. From the view of the time-frequency window, some common principles to be complied for choosing a wavelet function are presented for actual application, also, the amplitudefrequency characteristic and phase-frequency characteristic in frequency domain of common wavelet functions are studied. Deep study has been done on the specifically of the rotor fault characteristic component in start-up period of asynchronous motors, the wavelet ridge detection method of faulted rotor in start-up period is presented, so the accurate diagnosis of such fault is realized. It is demonstrated by the actual analysis that the new detection method has no special demand on the load quantity of the motor [8]. Even for a motor which runs with null load, the faulted rotor with only one broken bar can be detected accurately. The reasons of high misdiagnosis rate of faulted rotor under steady running state have been intensively studied, and the relevant improved methods have been put forward, moreover, the simultaneous monitoring of stator 
voltage signal is also included into the detection of faulted rotor under steady condition. On the foundation above, a novel method based on frequency-distance comparing to detect rotor fault is presented. It is the first time that wavelet analysis has been introduced into the online detection area of faulted motor rotor under steady state, and the wavelet algorithm to detect the rotor fault characteristic is presented [9]. As the result, the diagnosis accuracy has been improved greatly. A novel wavelet analysis method on the basis of the symmetry measurement of three-phase current is presented to detect the incipient inter-turn shorts fault of the asynchronous motor. Since wavelet transform is an analysis method based on frequency band extraction, it shows itself excellent robust against the frequency disturbance of the power net. Wavelet transform modulus maximum method is discussed at large to detect the sudden change signal of the stator windings fault. The analysis shows that wavelet transform modulus maximum method can not only detect the sudden change signal when a fault occurs in the stator windings, but can localize the time point when the sudden change happens.

An in-depth analysis is performed to study the frequency subband criss-cross phenomenon which comes about in signal decomposition with wavelet packet, and the reason is found to be the continuous signal decomposition with $\mathrm{W}$ 。 used by wavelet packet and frequency spectra periodicity of the corresponding low and high filters [10]. Based on this work, a general expression is given to decide the sequence of subband at the same level from low to high frequencies. Wavelet packet method is presented to detect the incipient axis fault, and research work on the abnormal axis vibration signal's decomposition and reconstruction techniques of wavelet packet is performed. It is demonstrates by the research that the high-frequency characteristic component of the abnormal axis vibration signal can be effectively extracted with wavelet packet technique, which is important to detect the incipient axis faults of asynchronous motor.

Research work has gone deep into signals denoising of the faulted asynchronous motor using wavelet and wavelet packet analysis, and denoising approach based on the best wavelet packet base is proposed. Wavelet packet method is more delicate compared with wavelet method. However, using best wavelet packet base to perform signal decomposition produces wavelet packet coefficients between which difference is more evident. The research work demonstrates that using best wavelet packet base to decompose signal will perform best denoising effect. Two sets of designs of wavelet intelligent diagnosis system of incipient faults of asynchronous motor are put forward, and the wavelet intelligent diagnosis system based on digital signal processing (DSP) technique has been successfully developed. The realization of such an system enrich the actual application contents of wavelet theory and lay a good foundation for further application of wavelet theory in power system.

\section{RESEARCH OF FAULT DIAGNOSIS OF THE WAVELET ANALYSIS}

The malfunction of the motor fault signal often contains a lot of time varying, short-term shocks, and composition. The traditional signal analysis methods, such as Fourei transform is powerless. In Fourei transformation, motor fault characteristics can not be effectively extracted. And when the test signal is non-stationary signal in the field of the motor test, Fourier transform can't get effective results. Wavelet transform as a time-frequency analysis method, it has the ability of denoting local signal characteristics in the time-frequency domain, at the same time it can pass the time and frequency window of flexible transformation to highlight the different frequency components of signals. Considering the advantages of wavelet transform processing non-stationary signal, wavelet transform is applied to the motor fault diagnosis and test in this paper.

Such as Fourier Transform and Wavelet Transform, the signal analysis theories are stated. The non-stationary and stationary signals of the motor are de-noised by FFT algorithm, Wavelet Transform, Mallat algorithm and Wavelet Packet Transform. As Wavelet Packet Transform has transfer feature to useful signals and restraint feature to noise, just as a band-pass filter, it is most suitable for removing the noise from non-stationary and stationary signals, which is proved effectively by the theory analysis and the comparison results.

The wavelet Packet Transform has time-frequency feature and multi-resolution feature [11]. Not only the whole signal but the partial signal can be analyzed, so the fault feature of non-stationary transient signals can be caught correctly. As the vibration signals and current signals of the motor can reflect the early fault, the fault diagnosis of the motor can be achieved by the vibration and current signal. The mechanism of the motor and fault feature frequency of the vibration signals and the current signals which are summarized. The feature extraction of the vibration and current signals is processed based on Wavelet Packet analysis. The fault of the motor is made by man to simulate true fault. And the vibration and current signals are sampled in the corresponding conditions. After that the normal and fault sampled signals are analyzed and compared, the Wavelet Packet decomposition and reconstruction of the normal and fault signals are then processed. The characteristic frequency branches are extracted from the reconstruction signals. The motor fault is identified through the comparison of the normal and fault characteristic frequency branches. In this thesis the fault is diagnosed and the fault feature is extracted by the changes of the feature frequency components. Great differences are found between normal and fault signals through the quantitative comparison results of the energy eigenvalue. Furthermore, Which Wavelet analysis is proved, fault diagnosis of asynchronous motors can be achieved correctly .

\section{CONCLUSIONS}

With the development of modern science and technology, electric machines play a more and more important role in modern industrial plants. The risk of machine failing can be remarkably reduced if normal service conditions can be arranged in advance. In other 
words, one may avoid very costly expensive downtime of plant by proper time scheduling of machine replacement or repair if warning of impeding failure can be obtained in advance. In this article,the working principle of the asynchronous motor and its common failure forms are analyzed and summarized.On this basis, the wavelet change of the application of the asynchronous motor that is carried out 。 In normal state and failure state, the motor vibration and current signal was reconstruct of the wavelet packet decomposition and single, and the fault characteristic frequency band that is conducted to extract.The characteristics of the normal operation and fault run time spectrum is carried on the detailed comparison. Based on the wavelet packet decomposition and reconstruction of motor fault feature extraction method can effective characterization of failure from the characteristic spectrum reconstruction signal waveform 。 Thus proves that the method based on wavelet analysis can accurately implement motor fault diagnosis.

\section{REFERENCES}

[1] Li Huabin. mechanical characteristics of three-phase asynchronous motors, brake theory [J]. 2011 scientific and technical information, (19): 524.
[2] J. M. ANJOS, G. K. CORACINI, E. VILLANI. A proposal and verification of a software architecture based on Labview for a multifunctional robotic end-effector[J]. Advances in Engineering Software, 2013, (55): 32-44.

[3] H. Krim, D. Tucker, S. Mallat, D. Donoho, On Denoising and Best Signal Representation, IEEE Trans. on Information Theory, 2011, 45(7), 2225-2238.

[4] J.F. Watson. Detection in N.C. Paterson. Improved Techniques for Rotor Fault

[5] Three-phase Induction Motors. IEEE Industry Applications Conference, 2008, vol 271-277.

[6] Jinzhu, Wang Wanyou. fault diagnosis and maintenance of mechanical and electrical equipment $[\mathrm{M}]$. Beijing: chemical industry press. 2010

[7] Wen Huixia. discussion on mechanical characteristics of threephase asynchronous motors, starting, braking and speed [J] Science and technology innovation Herald, 2011 (27): 71 74.

[8] Liu Zilin. motors and electrical control [M]. Beijing: Publishing House of electronics industry 2008.

[9] X. L. WEN, R. X. GUO. based on Labview virtual panel[J].Design of electronic power network frequency measurement system Energy Procedia, 2012, 17(1):456-461.

[10] Guo Xijin, Xu Jinxia, Kong Lili. A new method of asynchronous motor fault diagnosis[J].Industry and Minc Automation. 2014,40(3):60-63.

[11] Chen Jie. Photoelectric conversion in PN junction of high-pure Ptype silicon [J]. Semiconductor Optoelectronics 2008,19(3):208 209. 